\title{
Time-optimal synthesis for three relevant problems: the Brockett integrator, the Grushin plane and the Martinet distribution
}

\author{
Davide Barilari, Ugo Boscain, Enrico Le Donne, Mario Sigalotti
}

\begin{abstract}
We construct the time-optimal synthesis for 3 problems that are linear in the control and with polytopic constraints in the controls. Namely, the Brockett integrator, the Grushin plane, and the Martinet distribution. The main purpose is to illustrate the steps in solving an optimal control problem and in particular the use of second order conditions. The Grushin and the Martinet case are particularly important: the first is the prototype of a rank-varying distribution, the second of a non-equiregular structure.
\end{abstract}

\section{INTRODUCTION}

Constructing a time-optimal synthesis for problems that are affine in the controls and have polytopic constraints in the controls

$\dot{p}=F_{0}(p)+\sum_{i=1}^{m} u_{i} F_{i}(p), \quad u_{1}, \ldots, u_{n} \in[-1,1], \quad p(0)=p_{0}$

is a difficult and challenging problem. Usually the steps are the following

- STEP 1: FIRST ORDER NECESSARY CONDITIONS. In optimal control, the first order necessary conditions for optimality are given by the celebrated Pontryagin Maximum Principle [PBGM83] (PMP for short) that extends the Euler-Lagrange equations of calculus of variations to problems with non-holonomic constraints. The PMP restricts the set of candidate optimal trajectories starting from $p_{0}$ to a family of trajectories, called extermals, parameterised by a covector $\lambda(0) \in T_{p_{0}}^{*} M$.

- STEP 2. HIGHER ORDER CONDITIONS. These conditions are used to restrict further the set of candidate optimal trajectories.

- STEP 3. SELECTION OF THE OPTIMAL TRAJECTORIES. One should check that each extremal starting from $p_{0}$ does not intersect another extremal (starting from $p_{0}$ ) having a smaller cost at the intersection point. Even if the techniques described above are very powerful, in general computing explicitly an optimal synthesis is hard and the complexity grows quickly with the dimension of the space. The main difficulties are:

- the integration of the Hamiltonian equations given by the PMP (which in general is not integrable, unless there are many symmetries);

Université Paris Diderot, IMJ-PRG, UMR CNRS 7586 - UFR de Mathématiques, France, davide.barilari@imj-prg.fr

Ugo Boscain, CNRS, CMAP, École Polytechnique, Palaiseau, France, \& Team GECO, INRIA Saclay, ugo.boscainapolytechnique.edu

Department of Mathematics and Statistics, P.O. Box 35, FI-40014, University of Jyväskylä, Finland, ledonne@msri.org

INRIA Saclay, Team GECO \& CMAP, École Polytechnique, Palaiseau, France, mario.sigalottieinria.fr
- the characterisation of singular trajectories (which are extremals corresponding to controls that are not given directly by the maximum condition of the PMP);

- the verification of higher order conditions;

- the selection of optimal trajectories. This is the most difficult step since the comparison should be done not only among extremals that are close one to the other, but among all of them. The problem is indeed global.

For these reasons, the construction of optimal syntheses is already challenging in dimension 2 [BP04] and few examples have been solved in dimension 3 [SL96].

In this paper we show that a careful use of the steps mentioned above and in particular a careful use of higher order conditions permits to solve some important problems as the Brockett integrator, the Grushin plane and the Martinet distribution. Beside the well known Brockett integrator (also known as the Heisenberg group) for which the time optimal synthesis was constructed in [BLD13] with different techniques, the Grushin and Martinet case are very important. Indeed, the Grushin case is the simplest example of control problem where the dimension of the admissible velocities could drop down and the Martinet example is the simplest example in which the number of brackets necessary to get the Lie-bracket generating condition varies with the point. They appeared often in the literature as prototypes of more complicated systems. They where deeply studied when controls are bounded on the disk. However to our knowledge, the case with controls bounded in a polytope is new.

\section{Problem Setting And CONNection with Finsler GEOMETRY}

We study in this paper time-optimal control problems of the following type. Let $M$ be a smooth manifold, $k \in \mathbb{N}$ and take $X_{1}, \ldots, X_{k}$ in the space $\operatorname{Vec}(M)$ of smooth vector fields on $M$. Assume that $X_{1}, \ldots, X_{k}$ satisfy the Lie bracket generating condition $\left(\operatorname{Lie}\left(X_{1}, \ldots, X_{k}\right)\right)_{p}=T_{p} M$ for all $p \in M$. Here, given a family $\mathcal{F}$ of vector fields, we denote by $\operatorname{Lie}(\mathcal{F})$ and $\mathcal{F}_{p}$ the Lie algebra generated by $\mathcal{F}$ and the evaluation of the elements of $\mathcal{F}$ at a point $p$, respectively. We consider the problem of minimizing the time $T \geq 0$ for which there exist $p:[0, T] \rightarrow M$ absolutely continuous and $u:[0, T] \rightarrow \mathbb{R}^{k}$ measurable such that

$$
\left\{\begin{array}{l}
\dot{p}(t)=u_{1}(t) X_{1}(p(t))+\cdots+u_{k}(t) X_{k}(p(t)), \\
u(t) \in[-1,1]^{k} \\
p(0)=p, \quad p(T)=q .
\end{array}\right.
$$

The condition $u(t) \in[-1,1]^{k}$ can be rewritten as $|u(t)|_{\infty} \leq 1$, using the notation $|\cdot|_{\infty}$ for the max norm in 
$\mathbb{R}^{k}$. If we replace such a constraint by $|u(t)| \leq 1$ (with $|\cdot|$ the Euclidean norm), the value function of the problem would be the sub-Riemannian distance for the sub-Riemannian structure for which $X_{1}, \ldots, X_{k}$ is an orthonormal basis of the corresponding distribution. In this sense we are considering here a sub-Finsler problem, whose solutions are sub-Finsler geodesics.

\section{A. Hamiltonian formalism and Pontryagin Maximum Prin- ciple}

If a pair $(p(t), u(t))$ is a time minimizer for (1), then it satisfies the first order necessary conditions given by the Pontryagin Maximum Principle (PMP).

Define the Hamiltonian

$$
\mathcal{H}(\lambda, p, u):=\langle\lambda, f(p, u)\rangle=\sum_{i=1}^{k} u_{i}\left\langle\lambda, X_{i}(p)\right\rangle,
$$

for $\lambda \in T_{p}^{*} M, p \in M$, and $u \in \mathbb{R}^{k}$. For every $u \in \mathbb{R}^{k}$, let $\overrightarrow{\mathcal{H}}(\cdot, \cdot, u)$ be the vector field on $T^{*} M$ uniquely determined by the relation

$$
\sigma(\cdot, \overrightarrow{\mathcal{H}}(\lambda, p, u))=d_{(\lambda, p)} \mathcal{H}(\lambda, p, u),
$$

where $\sigma$ is the canonical symplectic form on $T^{*} M$.

Define the maximized Hamiltonian

$$
H(\lambda, p)=\sum_{i=1}^{k}\left|\left\langle\lambda, X_{i}(p)\right\rangle\right|
$$

Theorem $1(P M P)$ : Let $(p(t), u(t))$ be a time minimizer for Problem (1). Then there exist an absolutely continuous function $\lambda:[0, T] \rightarrow T^{*} M$ and a constant $\lambda_{0} \geq 0$ such that

(i) $\lambda(t) \in T_{p(t)}^{*} M \backslash\{0\}$, for every $t \in[0, T]$,

(ii) the pair $(\lambda(t), p(t))$ satisfies the Hamiltonian equation

$$
(\dot{\lambda}(t), \dot{p}(t))=\overrightarrow{\mathcal{H}}(\lambda(t), p(t), u(t))),
$$

for almost every $t \in[0, T]$.

(iii) $\mathcal{H}(\lambda(t), p(t), u(t))=H(\lambda(t), p(t))=\lambda_{0}$, for almost every $t \in[0, T]$.

If $\lambda(\cdot), p(\cdot)$ satisfy for some $u(\cdot)$ and $\lambda_{0}$ the conditions (i), (ii), (iii) of Theorem 1 , we say that $(\lambda(\cdot), p(\cdot))$ is an extremal pair, that $p(\cdot)$ is an extremal trajectory, and that $\lambda(\cdot)$ is an extremal lift of $p(\cdot)$.

For every vector field $Y$, if $(\lambda(\cdot), p(\cdot))$ is an extremal pair, then the function $t \mapsto\langle\lambda(t), Y(p(t))\rangle$ is absolutely continuous and its derivative satisfies

$$
\frac{d}{d t}\langle\lambda(t), Y(p(t))\rangle=\left\langle\lambda(t), \sum_{j=1}^{k} u_{j}(t)\left[X_{j}, Y\right](p(t))\right\rangle,
$$

for almost every $t$.

\section{B. Second order optimality conditions}

Our aim is to recall necessary conditions for the optimality of an extremal trajectory whose corresponding control is piecewise constant. We refer to [AG90]. (See also [AS03], [Sig05].)

Theorem 2: Let $(p(\cdot), u(\cdot))$ be an extremal pair for Problem (1) and let $\lambda(\cdot)$ be an extremal lift of $p(\cdot)$. Assume that $\lambda(\cdot)$ is the unique extremal lift of $p(\cdot)$, up to multiplication by a positive scalar. Assume that there exist $0=\tau_{0}<\tau_{1}<\tau_{2}<$ $\cdots<\tau_{K}<\tau_{K+1}=T$ and $u^{0}, \ldots, u^{K} \in \mathbb{R}^{k}$ such that $u(\cdot)$ is constantly equal to $u^{j}$ on $\left(\tau_{j}, \tau_{j+1}\right)$, with $j=0, \ldots, K$.

Fix $j=1, \ldots, K$. Let $Y_{i}=f\left(\cdot, u^{i}\right) \in \operatorname{Vec}(M)$, for all $i=0, \ldots, K$. Recursively define the following operators $P_{j}=P_{j-1}=\operatorname{id}_{\operatorname{Vec}(M)}$,

$$
\begin{gathered}
P_{i}=P_{i-1} \circ e^{\left(\tau_{i}-\tau_{i-1}\right) \operatorname{ad}\left(Y_{i-1}\right)}, \quad \forall i \in\{j+1, \ldots, K\}, \\
P_{i}=P_{i+1} \circ e^{-\left(\tau_{i+2}-\tau_{i+1}\right) \operatorname{ad}\left(Y_{i+1}\right)}, \quad \forall i \in\{0, \ldots, j-2\} .
\end{gathered}
$$

Define the vector fields

$$
Z_{i}=P_{i}\left(Y_{i}\right), \quad \forall i \in\{0, \ldots, K\} .
$$

Let $Q$ be the quadratic form

$$
Q(\alpha)=\sum_{0 \leq i<l \leq K} \alpha_{i} \alpha_{l}\left\langle\lambda\left(\tau_{j}\right),\left[Z_{i}, Z_{l}\right]\left(p\left(\tau_{j}\right)\right)\right\rangle,
$$

defined on the space

$$
W=\left\{\alpha \in \mathbb{R}^{K+1} \mid \sum_{i=0}^{K} \alpha_{i}=0, \sum_{i=0}^{K} \alpha_{i} Z_{i}\left(p\left(\tau_{j}\right)\right)=0\right\} .
$$

If $Q$ is not negative semi-definite, i.e., if there exists $\alpha \in W$ such that $Q(\alpha)>0$, then $p(\cdot)$ is not time-minimizing.

\section{Switching functions, singular, abnormal, and regular arcs}

With every extremal pair $(\lambda(\cdot), p(\cdot))$, for each $j=1, \ldots, k$ we associate the switching functions

$$
t \mapsto \varphi_{j}(t):=\left\langle\lambda(t), X_{j}(p(t))\right\rangle .
$$

By formula (4) we have that

$$
\dot{\varphi}_{j}(t)=\left\langle\lambda(t), \sum_{i=1}^{k} u_{j}(t)\left[X_{i}, X_{j}\right](p(t))\right\rangle .
$$

The maximality condition (iii) of the PMP and (3) imply that

$$
\left|\varphi_{1}(t)\right|+\cdots+\left|\varphi_{k}(t)\right|=\lambda_{0}, \quad \text { for all } t
$$

and that, for all $j=1, \ldots, k$ and almost all $t$,

$$
\varphi_{j}(t) \neq 0 \Longrightarrow u_{j}(t)=\operatorname{sign} \varphi_{j}(t) .
$$

The restriction of an extremal pair $(\lambda(\cdot), p(\cdot))$ to some open nonempty interval $I \subset[0, T]$ is called

(i) an abnormal arc if $\varphi_{j}(t) \equiv 0$ on $I$ for all $j=1, \ldots, k$;

(ii) a $\varphi_{j}$-singular arc if $\varphi_{j}(t) \equiv 0$ on $I$;

(iii) a regular $\operatorname{arc}$ if $\varphi_{j}(t) \neq 0$ for every $t \in I$ and for every $j=1, \ldots, k$.

(iv) a bang arc if the control $u(\cdot)$ associated with the trajectory is constant and takes values in $\{1,-1\}^{k}$.

Notice that a regular arc is a bang arc, but the converse is not true. Indeed, bang arcs can be singular (see Section V).

A bang-bang trajectory is a curve corresponding to a control that is piecewise constant with values $\{1,-1\}^{k}$. In particular, a concatenation of regular arcs is a bang-bang trajectory, called regular bang-bang trajectory. 
Remark 3: An arc is abnormal if and only if it is $\varphi_{j-}$ singular for all $j=1, \ldots, k$ and if and only if $\lambda_{0}=0$. The latter equivalence follows from (8). In particular, if a trajectory contains an abnormal arc then the whole trajectory is an abnormal arc.

\section{BROCKETT INTEGRATOR}

In this section we provide a description of the timeminimizing trajectories for the Brockett integrator called also the Heisenberg group. The same results have been previously obtained in [BLD13] using methods of metric geometry. The aim of this section is to illustrate how to exploit the geometric-control tools presented above to recover such results.

We consider Problem (1) on the Heisenberg group $\mathbb{H} \simeq \mathbb{R}^{3}$ determined by the vector fields

$$
X_{1}=\partial_{x}-\frac{y}{2} \partial_{z}, \quad X_{2}=\partial_{y}+\frac{x}{2} \partial_{z} .
$$

Let us introduce the vector field $X_{3}=\partial_{z}$, which satisfies $\left[X_{1}, X_{2}\right]=X_{3}$ and $\left[X_{1}, X_{3}\right]=\left[X_{2}, X_{3}\right]=0$.

We use the notation from the previous section. Formula (7) gives immediately

$$
\dot{\varphi}_{1}=-u_{2} \varphi_{3}, \quad \dot{\varphi}_{2}=u_{1} \varphi_{3}, \quad \dot{\varphi}_{3}=0,
$$

where $\varphi_{3}(t)=\left\langle\lambda(t), X_{3}(p(t))\right\rangle$.

In the next sections we characterize the abnormal, singular, and regular arcs for the associated time-optimal control problem.

\section{A. Abnormal arcs}

Lemma 4: The only abnormal arcs on $\mathbb{H}$ are the constant curves.

Proof: From Remark 3, we have $\varphi_{1}(t)=\varphi_{2}(t)=0$ for all $t$. By non-triviality of the covector $\lambda(\cdot)$, we deduce that $\varphi_{3}(t) \neq 0$ for every $t$. By the first two equations in (11), we get $u_{1}(t)=u_{2}(t)=0$ for almost every $t$.

\section{B. Singular arcs}

Lemma 5: On $\mathbb{H}$ the nonconstant trajectories that have singular arcs are exactly those for which there exists $j \in$ $\{1,2\}$ such that $u_{j}$ is constantly equal to 1 or -1 . All of them consist of a single singular arc and are time minimizers.

Proof: In what follows the roles of $u_{1}$ and $u_{2}$ are symmetric. Consider a nontrivial extremal trajectory that is $\varphi_{1}$-singular when restricted to an interval $I$, i.e., $\varphi_{1} \equiv 0$ on $I$. Because of Lemma 4, the trajectory does not have abnormal arcs, i.e., $\lambda_{0} \neq 0$. Hence, by (8), $\varphi_{2}$ never vanishes on $I$. By (9), $u_{2}$ is constantly equal to 1 or -1 on $I$. From the first equation in (11) we have $\varphi_{3}=0$ on $I$, and hence on the whole interval of definition of the trajectory. In particular, by (11) we have that the whole trajectory is $\varphi_{1}$-singular.

Conversely, every trajectory corresponding to $u_{2}= \pm 1$ constant and $u_{1}$ measurable with $\left|u_{1}\right| \leq 1$ has a $\varphi_{1}$-singular extremal lift with $\varphi_{2}=1$ and $\varphi_{1}=\varphi_{3}=0$.

Moreover, each such curve $\bar{p}=(\bar{x}, \bar{y}, \bar{z}):[0, T] \rightarrow \mathbb{H}$ is time-minimizing since $T=|\bar{y}(0)-\bar{y}(T)|$ and $|\dot{y}| \leq 1$ for every trajectory of $\dot{p}=u_{1} X_{1}(p)+u_{2} X_{2}(p)$ with $\left|u_{1}\right|,\left|u_{2}\right| \leq$ 1.

\section{Regular arcs}

Lemma 6: On $\mathbb{H}$ the trajectories that have a regular arc are regular bang-bang. Moreover, all arcs have the same length $s$ except possibly the last and the first arc, whose lengths are less than or equal to $s$. At the junction between regular arcs the components $u_{1}$ and $u_{2}$ of the control switch sign alternately.

Proof: Let $I$ be an interval on which the trajectory forms a regular arc. Without loss of generality, $\varphi_{1}, \varphi_{2}>0$ on $I$. Hence, by (9) we have $u \equiv(1,1)$ on $I$. Fix $t_{0} \in I$.

Two cases are possible:

(a) Assume $\varphi_{3}\left(t_{0}\right)=0$. By (11) we have that $\varphi_{1}$ and $\varphi_{2}$ are constant along the entire trajectory, which is then a single regular arc.

(b) Assume $\varphi_{3}\left(t_{0}\right) \neq 0$. Denote by $a$ the constant value of $\varphi_{3}$. Using (11) we find

$$
\varphi_{1}(t)=\varphi_{1}\left(t_{0}\right)-a\left(t-t_{0}\right), \quad \varphi_{2}(t)=\varphi_{2}\left(t_{0}\right)+a\left(t-t_{0}\right) .
$$

Without loss of generality $a>0$. Set $t_{1}=t_{0}+\varphi_{1}\left(t_{0}\right) / a$. If the trajectory is defined up to time $t_{1}$, then $\varphi_{1}$ and $\varphi_{2}$ are positive in the interval $\left(t_{0}, t_{1}\right)$ and $\varphi_{1}\left(t_{1}\right)=0$.

Since $u_{2}=1$ in a neighborhood of $t_{1}$, we deduce that $\varphi_{1}$ is affine in a neighborhood of $t_{1}$, with slope $-a$. Hence $\varphi_{1}<0<\varphi_{2}$ in a right-neighborhood of $t_{1}$. Then $t_{1}$ is the starting time of another regular arc with control $u=(-1,1)$.

Repeating this argument, backwards in time as well, we conclude that the extremal trajectory is the concatenation of regular arcs of length $\varphi_{2}\left(t_{1}\right) / a=\left(\varphi_{1}\left(t_{0}\right)+\varphi_{2}\left(t_{0}\right)\right) / \varphi_{3}\left(t_{0}\right)$, except possibly for the first and last arc, see Figure 1. The switching occur alternately for $u_{1}$ and $u_{2}$.

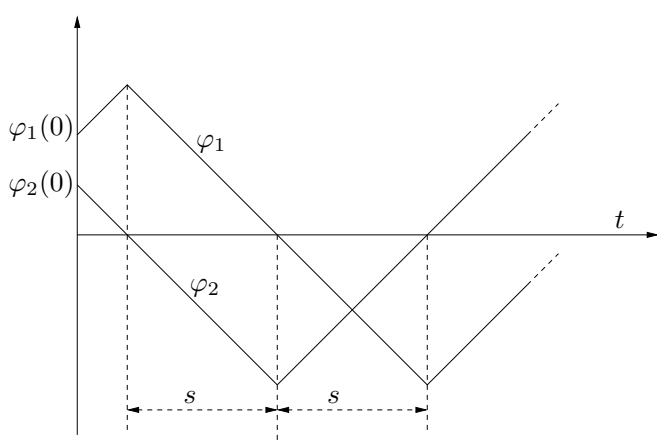

Fig. 1: The switching functions for the Brockett integrator, when $\varphi_{3} \neq 0$.

Let us mention that, up to reflection with respect to the $t$-axes, that amount to change the sign of $\varphi_{3}$, and a time shifting, these are all the possible cases when $\varphi_{3} \neq 0$.

\section{Bound on number of optimal regular arcs}

Proposition 7: A regular bang-bang trajectory with more than 5 arcs is not optimal.

Proof: Let us consider a trajectory with 6 bang arcs. By Lemma 6, without loss of generality we can assume that the successive values of the control are

$$
(-1,-1),(-1,1),(1,1),(1,-1),(-1,-1),(-1,1) \text {. }
$$


Denote the length of the internal bang arcs by $s$ (recall that the length of the arcs are the same, except possibly the first and last).

We are going to apply Theorem 2 by taking $j=3$. Let $\tau_{3}$ be the third switching time. Since at $\tau_{3}$ the function $\varphi_{2}$ switches sign, we have that $\varphi_{2}\left(\tau_{3}\right)=0$.

Up to multiplication of $\lambda(\cdot)$ by a positive scalar, we can normalize $\varphi_{3}$, which is constant, to -1 . Hence, $\varphi_{1}\left(\tau_{3}\right)=$ $s$, which implies that $\lambda(\cdot)$ is uniquely determined by the sequence of switching times. Set

$$
X_{+}=X_{1}+X_{2}, \quad X_{-}=X_{1}-X_{2} .
$$

We have

$$
\begin{aligned}
& Z_{0}=e^{-s \operatorname{ad}\left(X_{+}\right)} e^{s \operatorname{ad}\left(X_{-}\right)}\left(-X_{+}\right)=-X_{+}-2 s X_{3}, \\
& Z_{1}=e^{-s \operatorname{ad}\left(X_{+}\right)}\left(-X_{-}\right)=-X_{-}-2 s X_{3}, \\
& Z_{2}=X_{+}, \quad Z_{3}=X_{-}, \\
& Z_{4}=e^{s \operatorname{ad}\left(X_{-}\right)}\left(-X_{+}\right)=-X_{+}-2 s X_{3}, \\
& Z_{5}=e^{s \operatorname{ad}\left(X_{-}\right)} e^{s \operatorname{ad}\left(-X_{+}\right)}\left(-X_{-}\right)=-X_{-}-2 s X_{3} .
\end{aligned}
$$

A simple calculation shows that

$$
\begin{aligned}
& \sigma_{01}=\sigma_{05}=\sigma_{12}=\sigma_{23}=\sigma_{34}=\sigma_{45}=2, \\
& \sigma_{02}=\sigma_{04}=\sigma_{13}=\sigma_{15}=\sigma_{24}=\sigma_{35}=0, \\
& \sigma_{03}=\sigma_{14}=\sigma_{25}=-2 .
\end{aligned}
$$

Decomposing the relation $\sum_{i=0}^{5} \alpha_{i} Z_{i}\left(p\left(\tau_{3}\right)\right)=0$ on the basis $\left\{X_{+}\left(p\left(\tau_{3}\right)\right), X_{-}\left(p\left(\tau_{3}\right)\right), X_{3}\left(p\left(\tau_{3}\right)\right)\right\}$, and solving in $\alpha_{0}, \alpha_{1}, \alpha_{2}$, gives

$$
\alpha_{3}=-\alpha_{2}, \quad \alpha_{4}=-\alpha_{0}+\alpha_{2}, \quad \alpha_{5}=-\alpha_{1}-\alpha_{2} .
$$

Notice that the relation $\sum_{i=0}^{5} \alpha_{i}=0$ is automatically satisfied. Then we can parameterize the space $W$ appearing in the statement of Theorem 2 by $\alpha=\left(\alpha_{0}, \alpha_{1}, \alpha_{2}\right)$, i.e.,

$$
W=\left\{\left(\alpha_{0}, \alpha_{1}, \alpha_{2},-\alpha_{2},-\alpha_{0}+\alpha_{2},-\alpha_{1}-\alpha_{2}\right) \mid \alpha \in \mathbb{R}^{3}\right\},
$$

and write the quadratic form $Q$ as

$$
Q(\alpha)=4 \alpha_{0} \alpha_{1}+4 \alpha_{0} \alpha_{2}-4 \alpha_{2}^{2} .
$$

In particular, $Q(1,1,0)=4>0$, which implies that the trajectory is not optimal.

\section{E. Optimal trajectories and shape of the unit ball}

Here we summarize the results obtained in the previous sections and we plot the unit ball for the Brockett integrator.

Recall that once we characterize the controls $u_{1}(t)$ and $u_{2}(t)$ associated with an extremal trajectory, to recover the trajectory itself it is sufficient to solve the differential equation $\dot{\gamma}(t)=u_{1}(t) X_{1}(\gamma(t))+u_{2}(t) X_{2}(\gamma(t))$, i.e.,

$$
\left\{\begin{array}{l}
\dot{x}=u_{1}, \\
\dot{y}=u_{2}, \\
\dot{z}=\frac{1}{2}\left(u_{2} x-u_{1} y\right) .
\end{array}\right.
$$

In particular, the trajectory is determined by its projection onto the $x y$-plane, since the $z$ coordinate of the trajectory can be found by integration. As it is well-known, it computes the

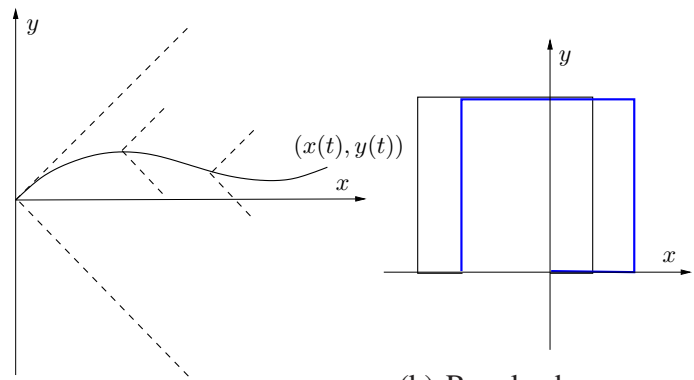

(a) Singular arcs

(b) Regular bang arcs

Fig. 2: Singular and regular arcs for the Brockett integrator

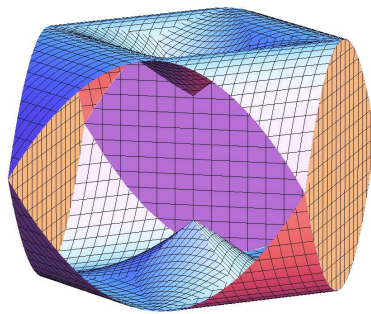

(a) Sphere

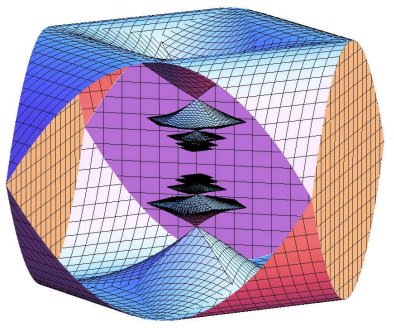

(b) Front
Fig. 3: Sphere and front for the Brockett integrator

signed area defined by the closed curve given by following $\gamma$ and then coming back to the origin along a line segment.

As discussed in Lemma 5, the singular trajectories correspond to $u_{1}(t)$ constantly equal to \pm 1 and $u_{2}(t)$ free (or the symmetric situation). In Figure $2 \mathrm{a}$ we can see an example of such a curve when $u_{1}(t)=1$. Recall that these curves are optimal for all times.

Regular bang-bang trajectories correspond to switching functions as in Figure 1, where the controls switch sign alternatively. These trajectories draw squares in the $x y$-plane as in Figure $2 \mathrm{~b}$. If such a trajectory has more than 5 bang arcs, then Proposition 7 guarantees that the trajectory is not optimal.

Notice that there are time-minimizing curves of this kind with 5 regular bang arcs, as illustrated in Figure 2b. However, not all bang-bang trajectories with 5 bang arcs are timeminimizing. Indeed, if such a square is swept more than once, then it is no more a minimizer. Finally, let us also remark that for every minimizer with 5 regular bang arcs there exists a minimizer with 4 regular bang arcs joining the same endpoints (see again Figure 2b).

Once the shape of optimal trajectories is known, a picture of the unit sphere for the Brockett integrator can be easily drawn. See Figures 3a. Figure 3b shows the so called unit front, i.e., the end point of all geodesics at time 1 .

\section{GRUSHIN STRUCTURE}

In this section we provide a description of the timeminimizing trajectories in the Grushin plane. The classical sub-Riemannian structure on the Grushin plane is the metric structure on $\mathbb{R}^{2}$ determined by the choice of the orthonormal 
vector fields

$$
X_{1}=\partial_{x}, \quad X_{2}=x \partial_{y} .
$$

Let us introduce the vector field $X_{3}=\partial_{y}$.

The Lie algebra generated by $X_{1}, X_{2}, X_{3}$ satisfies the same commutator relations as in the Heisenberg group, namely

$$
\left[X_{1}, X_{2}\right]=X_{3}, \quad\left[X_{1}, X_{3}\right]=\left[X_{2}, X_{3}\right]=0 .
$$

Thus identity (4) gives the equations for the switching functions along an extremal trajectory

$$
\dot{\varphi}_{1}=-u_{2} \varphi_{3}, \quad \dot{\varphi}_{2}=u_{1} \varphi_{3}, \quad \dot{\varphi}_{3}=0 .
$$

In particular, $\varphi_{3}$ is constant. From $X_{2}=x X_{3}$ we have the additional relation

$$
\varphi_{2}=x \varphi_{3}
$$

In the case $\varphi_{3}=0$, we get $\varphi_{2} \equiv 0$ and $\varphi_{1}$ equals a nonzero constant (otherwise the covector is identically zero). Reasoning as in Lemma 5, we have immediately the following result

Lemma 8: The nonconstant trajectories that have singular arcs are exactly those for which $u_{1}$ is constantly equal to 1 or -1 . All of them consist of a single singular arc and are time minimizers.

Let us then assume in what follows $\varphi_{3} \neq 0$.

Lemma 9: The only abnormal arcs are the constant curves contained in the set $\{x=0\}$. Consequently, no minimizer joining two distinct points is abnormal.

If $\varphi_{3} \neq 0$ and the trajectory is not abnormal, then as in Lemma 6 it is regular bang-bang, all arcs have the same length $s$ except possibly the last and the first arc, whose lengths are less than or equal to $s$. At the junction between bang arcs the components $u_{1}$ and $u_{2}$ of the control switch sign alternately.

Moreover, on a regular bang-bang trajectory, $u_{2}$ switches on the line $x=0$, since, if $\varphi_{2}(t)=0$ at a point $t$, then $x(t) \varphi_{3}=0$. Therefore if a bang-bang trajectory has an internal bang arc whose length is $s$, then $u_{1}$ switches on the lines $x= \pm s$. Moreover, we claim that, for trajectories with a single $u_{1}$-switch the function $u_{1}$ goes from 1 to -1 if the switch occurs in the half-plane $x>0$ while it goes from -1 to 1 in the half-plane $x<0$. Indeed,

$$
\begin{aligned}
\operatorname{sign}\left(\dot{\varphi}_{1}\right) & =-\operatorname{sign}\left(u_{2} \varphi_{3}\right)=-\operatorname{sign}\left(\varphi_{2} \varphi_{3}\right) \\
& =-\operatorname{sign}\left(x \varphi_{3}^{2}\right)=-\operatorname{sign}(x) .
\end{aligned}
$$

1) Bound on number of optimal regular arcs: Regarding optimality, we prove in this section the following lemma.

Lemma 10: A regular bang-bang trajectory with more than 3 arcs is not optimal. If, moreover, the trajectory starts on the $y$-axis and it is optimal, it has at most 2 arcs.

Contrarily to what happens for the Brockett integrator, the role of the two vector fields $X_{1}, X_{2}$ is not symmetric. The replacement of $\left(u_{1}, u_{2}\right)$ by $\left(-u_{1},-u_{2}\right)$ coupled with the reversion in the order of bangs, on the contrary, still yields a symmetric, equivalent, situation. This is a general fact, since it simply corresponds to reverse the parameterization of the curve. Looking at regular bang-bang trajectories (see Figure 5) one immediately recognises that the proof of the lemma can be given by looking at two types of bang-bang trajectories, whose successive values of the control are

$$
(1,-1),(1,1),(-1,1),(-1,-1)
$$

and

$$
(1,1),(-1,1),(-1,-1),(1,-1),
$$

respectively. In the first case, one notices that reflecting the second and third bang arcs with respect to the $y$-axis yields another horizontal curve with the same length, which is not extremal. Hence the curve is not optimal. This argument also shows that regular bang-bang trajectories starting from the $y$-axis and with more than 2 bang arcs are not optimal.

In the second case, let us apply Theorem 2 at the second switching time. One gets

$$
\begin{array}{ll}
Z_{0}=X_{1}+X_{2}+2 s X_{3}, & Z_{1}=-X_{1}+X_{2}, \\
Z_{2}=-X_{1}-X_{2}, & Z_{3}=X_{1}-X_{2}+2 s X_{3} .
\end{array}
$$

Parameterizing the space $W$ by the coordinates $\alpha_{0}, \alpha_{1}$ we get that $W=\left\{\left(\alpha_{0}, \alpha_{1},-\alpha_{1},-\alpha_{0}\right) \mid \alpha_{0}, \alpha_{1} \in \mathbb{R}\right\}$. Normalizing $\varphi_{3}=1$ (uniqueness of the covector up to a positive factor is proved as in the case of the Brockett integrator), we write the quadratic form $Q$ as

$$
Q\left(\alpha_{0}, \alpha_{1}\right)=2 \alpha_{0}^{2}+4 \alpha_{0} \alpha_{1}-2 \alpha_{1}^{2} .
$$

Since $Q(1,0)$ is positive, the considered trajectory is not optimal. This concludes the proof of Lemma 10.

2) Optimal trajectories and shape of the unit ball: In the Grushin plane we have singular trajectories that are similar to the ones obtained for the Brockett integrator, see Figure 4. Let us stress that in this case the tangent vector of the curve is forced to be inside a cone whose width increase with the $x$ coordinate. The picture of the regular bang bang trajectories

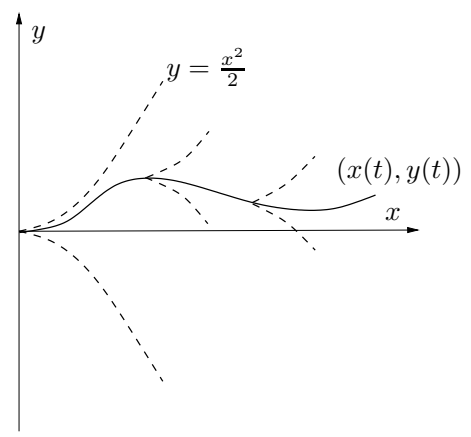

Fig. 4: Singular trajectories in the Grushin plane

is in Figure 5. These trajectories lose optimality as soon as they reach the vertical axes. The picture of the unit ball in the Grushin plane with this structure is in Figure 6. 


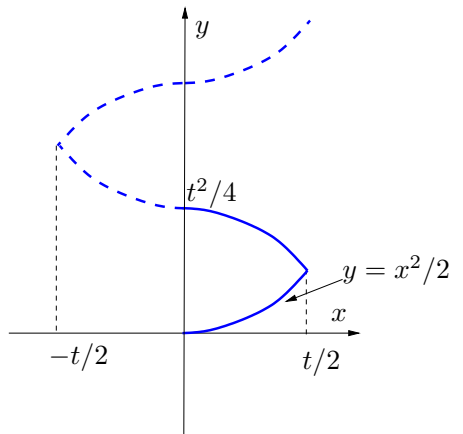

Fig. 5: Bang trajectories in the Grushin plane

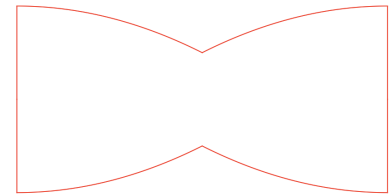

(a) Sphere (b) Front

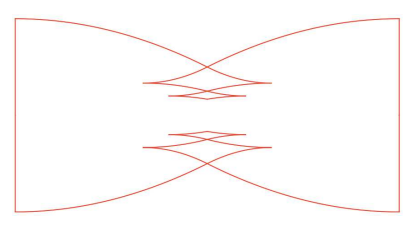

Fig. 6: Sphere in the Grushin structure

\section{MARTINet StRUCture}

In this section we provide a description of the timeminimizing trajectories for the Martinet structure. This is the easiest example where nontrivial abnormal minimizers appear.

The classical sub-Riemannian structure on the Martinet space is the metric structure on $\mathbb{R}^{3}$ determined by the choice of the orthonormal vector fields

$$
X_{1}=\partial_{x}+\partial_{y}+y^{2} \partial_{z}, \quad X_{2}=\partial_{x}-\partial_{y}+y^{2} \partial_{z} .
$$

Let us introduce the vector fields

$$
\begin{array}{r}
X_{3}=\left[X_{1}, X_{2}\right]=4 y \partial_{z}, X_{4}=\left[X_{1}, X_{3}\right]=4 \partial_{z}, \\
X_{5}=\left[X_{2}, X_{3}\right]=-4 \partial_{z} .
\end{array}
$$

The associated switching functions are

$$
\varphi_{i}(p, q)=\left\langle p, X_{i}(q)\right\rangle, \quad i=1, \ldots, 5 .
$$

They satisfy the system of differential equations

$$
\begin{array}{r}
\dot{\varphi}_{1}=-u_{2} \varphi_{3}, \quad \dot{\varphi}_{2}=u_{1} \varphi_{3}, \quad \dot{\varphi}_{3}=u_{1} \varphi_{4}+u_{2} \varphi_{5} \\
\dot{\varphi}_{4}=0, \quad \dot{\varphi}_{5}=0 .
\end{array}
$$

Remark 11: It follows from the bracket relations (16) that $\varphi_{4}$ and $\varphi_{5}=-\varphi_{4}$ are constants and we have $\varphi_{3}=y \varphi_{4}=$ $-y \varphi_{5}$. In particular, if $\varphi_{4}=0$, then $\varphi_{3}$ is also constantly equal to zero, and $\varphi_{1}, \varphi_{2}$ are constant.

Lemma 12: The nontrivial abnormal arcs are the horizontal lines contained in the plane $\{y=0\}$.

Proof: Assume that the trajectory is not reduced to a point and it is abnormal on some interval $I$. In particular we have $\varphi_{1}(t)=\varphi_{2}(t)=0$ for all $t \in I$, while its control $\left(u_{1}(t), u_{2}(t)\right)$ is not identically zero on $I$. From (17) one gets that $-u_{2}(t) \varphi_{3}(t)=u_{1}(t) \varphi_{3}(t)=0$. Hence, if we have $\varphi_{3}(t)=y(t) \varphi_{4}=0$ for every $t$ (recall that $\varphi_{4}$ is constant), then $y(t)=0$ for all $t \in I$, otherwise $\varphi_{4}=0$ and the covector is identically zero.

3) Singular arcs: Let us now consider a singular arc. We show that in this case we can recover its (singular) control by differentiation of the adjoint equations.

Indeed assume that the trajectory is $\varphi_{1}$-singular, i.e., $\varphi_{1} \equiv$ 0 on $I$, and we want to recover its associated control $u_{1}$. Notice that $\left|u_{2}\right|=1$ is constant and $\dot{\varphi}_{1}=-u_{2} \varphi_{3}$ is continuous, hence $\varphi_{1}$ is $C^{1}$ on $I$. Because $\dot{\varphi}_{1}(t) \equiv 0$, we have $\varphi_{3}(t) \equiv 0$ for all $t \in I$ (recall that $u_{2}$ is different from zero). We deduce that either $\varphi_{4}=0$ or $u_{1}=u_{2}$ on $I$. We have two possibilities:

(i) if $\varphi_{4}=0$ then $u_{1}$ is free;

(ii) if $\varphi_{4} \neq 0$ then $u_{1}=u_{2}$ and we obtain a special type of trajectory with bang arcs (the one that is horizontal), but there is no constraint in the length of the arc. Moreover one has $y=0$ on this arc.

The trajectories corresponding to singular arcs of type (i) coincide with those obtained for the Brockett integrator. The trajectories corresponding to singular arcs of type (ii) are of the same type as a regular bang arc, but with free time of switching (see below). The situation with $\varphi_{2}$-singular arcs is perfectly symmetric.

4) Regular arcs: Assume that both $\varphi_{1}(0), \varphi_{2}(0) \neq 0$. Because of Remark 11, we can assume that $\varphi_{4} \neq 0$ (otherwise the trajectory is made of a single bang arc). We want to show that

(a) If $\varphi_{1}(0) \varphi_{2}(0)>0$ then $\varphi_{1}, \varphi_{2}$ are linear in a left neighborhood of 0 .

(b) If $\varphi_{1}(0) \varphi_{2}(0)<0$ then $\varphi_{1}, \varphi_{2}$ are quadratic in a left neighborhood of 0 .

On a bang arc the controls satisfy $\left|u_{1}\right|=\left|u_{2}\right|=1$ and thus we can differentiate the identity (17) and get

$$
\begin{gathered}
\ddot{\varphi}_{1}=-u_{2} \dot{\varphi}_{3}=-4 u_{2}\left(u_{1}-u_{2}\right) \varphi_{4}, \\
\ddot{\varphi}_{2}=u_{1} \dot{\varphi}_{3}=4 u_{1}\left(u_{1}-u_{2}\right) \varphi_{4} .
\end{gathered}
$$

In case (a) we have that $u_{1}=u_{2}= \pm 1$, that implies $\ddot{\varphi}_{1}=$ $\ddot{\varphi}_{2}=0$. In case (b) we have $u_{1}-u_{2}= \pm 2$ and consequently $\ddot{\varphi}_{1}$ and $\ddot{\varphi}_{2}$ are constant and nonzero (recall that $\varphi_{4} \neq 0$ is constant). The equations for case (a) are

$$
\begin{gathered}
\varphi_{1}(t)=\varphi_{1}(0)+t \dot{\varphi}_{1}(0)=\varphi_{1}(0)-u_{2} \varphi_{3}(0) t, \\
\varphi_{2}(t)=\varphi_{2}(0)+t \dot{\varphi}_{2}(0)=\varphi_{2}(0)+u_{1} \varphi_{3}(0) t, \\
\varphi_{3}(t)=\varphi_{3}(0) .
\end{gathered}
$$

Notice that $\varphi_{3}(0)=y(0) \varphi_{4}$ is zero if we start on the abnormal set. The equations for case (b) are

$$
\begin{aligned}
\varphi_{1}(t)= & \varphi_{1}(0)-u_{2} \varphi_{3}(0) t-u_{2}\left(u_{1}-u_{2}\right) \varphi_{4} \frac{t^{2}}{2}, \\
\varphi_{2}(t)= & \varphi_{2}(0)+u_{1} \varphi_{3}(0) t+u_{1}\left(u_{1}-u_{2}\right) \varphi_{4} \frac{t^{2}}{2}, \\
& \varphi_{3}(t)=\varphi_{3}(0)+\left(u_{1}-u_{2}\right) \varphi_{4} t .
\end{aligned}
$$

In particular, the constant $\varphi_{4}$ determines the convexity of the quadratic arc of the switching functions. 
Lemma 13: A regular bang arc can enter in a singular arc only if the switching function is quadratic and has vanishing derivative at the switching point.

Proof: Assume, for instance, that at some time $t_{0} \in$ $I$ we have $\varphi_{1}\left(t_{0}\right)=1$ and $\varphi_{2}\left(t_{0}\right)=0$. Then the control $u_{1}(t)=\operatorname{sign} \varphi_{1}(t)$ is constantly equal to 1 in a neighborhood $U_{t_{0}}$ of $t_{0}$ and since $\varphi_{3}$ is continuous we deduce that $\dot{\varphi}_{2}=$ $u_{1} \varphi_{3}$ is also continuous in $U_{t_{0}}$. Since on the singular arc $\dot{\varphi}_{2}=0$, we conclude.

Let us assume that $\varphi_{1}(0)>0$ and $\varphi_{2}(0)<0$. In particular $\varphi_{1}$ and $\varphi_{2}$ are quadratic on a left neighborhood of 0 .

We are reduced to three possible cases for the the switching function $\varphi_{1}$ :

- it never vanishes in the quadratic part (we say that $\varphi_{1}$ is of type NI, for not intersecting),

- it vanishes in the quadratic part and is tangent to the zero level (type $T$ for tangent),

- it vanishes in the quadratic part and is transversal to the zero level (type I for intersecting).

In Figure 7 we picture the switching functions when $\varphi_{1}$ is of type NI, while Figures 8 and 9 correspond to type T and type I, respectively.

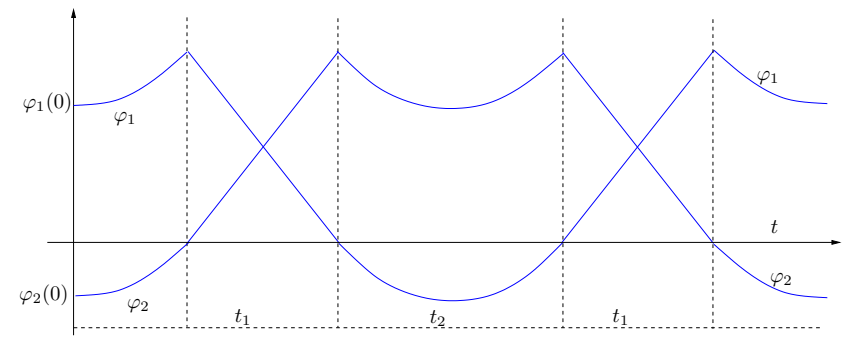

Fig. 7: Switching functions for the Martinet structure when $\varphi_{1}$ is of type NI.

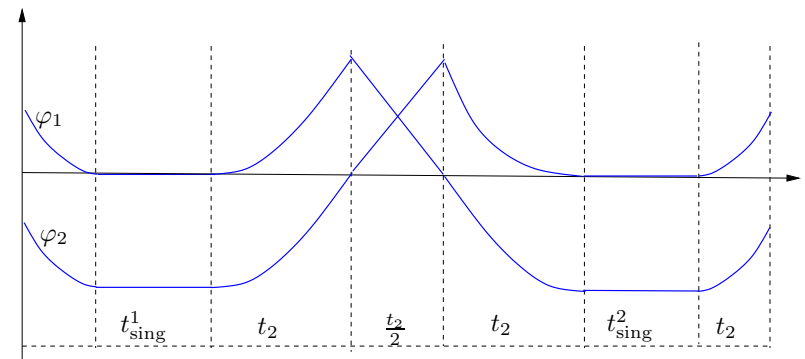

Fig. 8: Switching functions for the Martinet structure when $\varphi_{1}$ is of type T. The relation between the third and fourth bang arcs can be easily deduced from the expression of the switching functions.

Assuming that there are only regular bang arcs along the trajectory (as it is always the case when $\varphi_{1}$ is of type NI or I) we have the following result.

Proposition 14: The switching functions of a trajectory that has only regular bang arcs are periodic.

The proof of Proposition 14 is a simple consequence of the formulas of the switching functions and Lemma 13 . When $\varphi_{1}$

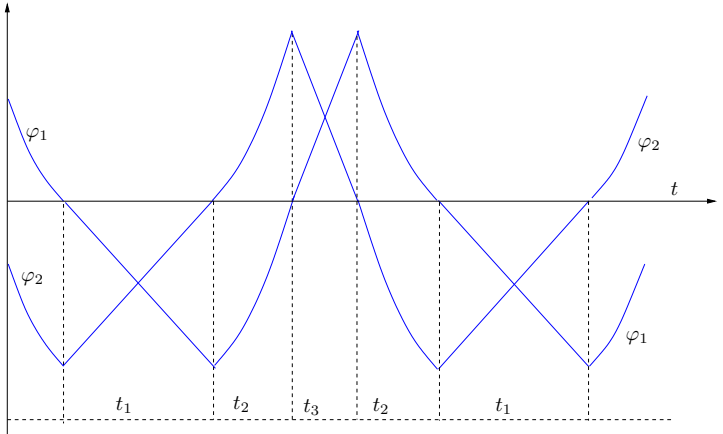

Fig. 9: Switching functions for the Martinet structure when $\varphi_{1}$ is of type I.

is of type T, the only freedom is in the length of singular arcs. The order in which the switching occur is as in Figures 7, 8 and 9 , up to the symmetry which sends $\left(\varphi_{1}, \varphi_{2}, \varphi_{3}, \varphi_{4}\right)$ into $\left(-\varphi_{1},-\varphi_{2}, \varphi_{3},-\varphi_{4}\right)$ (which corresponds to a reflection $y \rightarrow-y)$.

5) Bound on number of optimal regular arcs: The goal of this section is to prove the following result.

Proposition 15: A bang-bang trajectory with at least one regular arc and with more than 7 arcs (either bang or singular) is not optimal.

We distinguish in what follows trajectories for which the switching functions are of one of the three types NI, T, and I. In order to reduce the number of cases to be studied, we use the fact that time-reversion and reflection $y \rightarrow-y$ lead to trajectories with equivalent optimality properties.

Switching functions of type NI: We start by considering $\varphi_{1}$ of the type NI, as in Figure 7.

Lemma 16: A regular bang-bang trajectory of type NI with more than 5 arcs is not optimal.

Proof: We prove the first part of the lemma by showing that concatenations of the type

$$
(1,-1),(1,1),(-1,1),(1,1),(1,-1)
$$

are not optimal. All concatenations of 6 bang arcs, indeed, contain a concatenation of this type, up to symmetries.

For concatenations of type (18), applying Theorem 2 at the second switching time, we get by computations as the one seen in the previous sections that the space $W$ and the quadratic form $Q$ in the statement of Theorem 2 can be written as

$$
\begin{aligned}
W & =\left\{\left(\alpha_{0}, \alpha_{1}, 0,-\alpha_{1},-\alpha_{0}\right) \mid \alpha_{0}, \alpha_{1} \in \mathbb{R}\right\}, \\
Q\left(\alpha_{0}, \alpha_{1}\right) & =8\left(t_{1} \alpha_{0}^{2}+t_{2} \alpha_{0} \alpha_{1}\right) .
\end{aligned}
$$

Since $Q$ is not negative semidefinite, the corresponding trajectory is not optimal.

Switching functions of type T: We prove here the following result concerning trajectories corresponding to switching functions of the type $\mathrm{T}$ as in Figure 8.

Lemma 17: A trajectory of type $\mathrm{T}$ with more than 7 arcs is not optimal.

Proof: We first consider the situation where $t_{\text {sing }}^{k}>0$ for every $k$. We notice that every concatenations of 8 arcs 
contains, up to symmetries, a concatenation of $6 \operatorname{arcs}$ of the type

$$
(1,-1),(1,1),(-1,1),(-1,-1),(-1,1),(1,1) .
$$

We are going to show that a concatenation as in (19) is not optimal.

For concatenations of type (19), applying Theorem 2 at the third switching time (at which $y=0$ ), we get that the space $W$ and the quadratic form $Q$ in the statement of Theorem 2 are written as

$$
\begin{gathered}
W=\left\{\left(\alpha_{0}, \alpha_{1}, \alpha_{2},-\alpha_{0}, \alpha_{0}-\alpha_{2},-\alpha_{0}-\alpha_{1}\right) \mid \alpha \in \mathbb{R}^{3}\right\} \\
Q\left(\alpha_{0}, \alpha_{1}, \alpha_{2}\right)=2\left(t_{2}-2 t_{\text {sing }}^{2}\right) \alpha_{0}^{2}+8 t_{2} \alpha_{0} \alpha_{1}+ \\
8 t_{\text {sing }}^{2} \alpha_{0} \alpha_{2}-4 t_{\text {sing }}^{2} \alpha_{2}^{2} .
\end{gathered}
$$

Notice that $Q$ is not negative semidefinite, since $Q(\varepsilon, 1 / \varepsilon, 0)=2 \varepsilon^{2}\left(t_{2}-2 t_{\text {sing }}^{2}\right)+8 t_{2}>0$ for $\varepsilon$ small enough. Hence, the corresponding trajectory is not optimal.

In the case where $t_{\text {sing }}^{2}=0$, a concatenation as in (19) reduces to a concatenation of 4 bang arcs

$$
(1,-1),(1,1),(-1,1),(1,1) .
$$

Considering the following arc, we recover a concatenation as in (18), for which the same computations as in the previous section show non optimality.

Switching functions of type I: We consider here trajectories corresponding to switching functions of type I as in Figure 9. Notice that such trajectories never cross the plane $\{y=0\}$.

Lemma 18: A regular bang-bang trajectory of type I with more than 5 arcs is not optimal.

Proof: By the same symmetry considerations as in the cases NI and T, we are left to prove that concatenations of the type

$$
(1,-1),(-1,-1),(-1,1),(1,1),(1,-1),(-1,-1)
$$

and

$$
(-1,1),(1,1),(1,-1),(-1,-1),(-1,1),(1,1)
$$

are not optimal. Notice than in both cases the trajectory is contained in $\{y<0\}$.

The application of Theorem 2 to the two cases is very similar leading (computing the quadratic form $Q$ at the second switching time $\tau_{2}$ ) to the expressions

$$
\begin{aligned}
& Q\left(\alpha_{0}, \alpha_{1}, \alpha_{2}\right)=-4\left(t_{1}-t_{3}\right) \alpha_{0}^{2}-4 y\left(\tau_{2}\right) \alpha_{0} \alpha_{1} \\
& \quad-4\left(-2 t_{2}+2 t_{3}+y\left(\tau_{2}\right)\right) \alpha_{0} \alpha_{2}-4\left(2 t_{2}-t_{3}-y\left(\tau_{2}\right)\right) \alpha_{2}^{2}
\end{aligned}
$$

and

$$
\begin{aligned}
& Q\left(\alpha_{0}, \alpha_{1}, \alpha_{2}\right)=-4\left(t_{1}-t_{3}\right) \alpha_{0}^{2}-4 y\left(\tau_{2}\right) \alpha_{0} \alpha_{1} \\
& \quad-4\left(-2 t_{1}+2 t_{2}+y\left(\tau_{2}\right)\right) \alpha_{0} \alpha_{2}-4\left(t_{1}-2 t_{2}-y\left(\tau_{2}\right)\right) \alpha_{2}^{2}
\end{aligned}
$$

respectively. In both cases, since $y\left(\tau_{2}\right)<0$, one has that $Q(\varepsilon, 1 / \varepsilon, 0)=-4 y\left(\tau_{2}\right)+O\left(\varepsilon^{2}\right)$ is positive for $\varepsilon$ small enough. Theorem 2 then allows to conclude that the corresponding trajectories are not optimal.

By using the previously described optimality results one gets a picture of the unit ball as in Figure 10 and Figure 11.

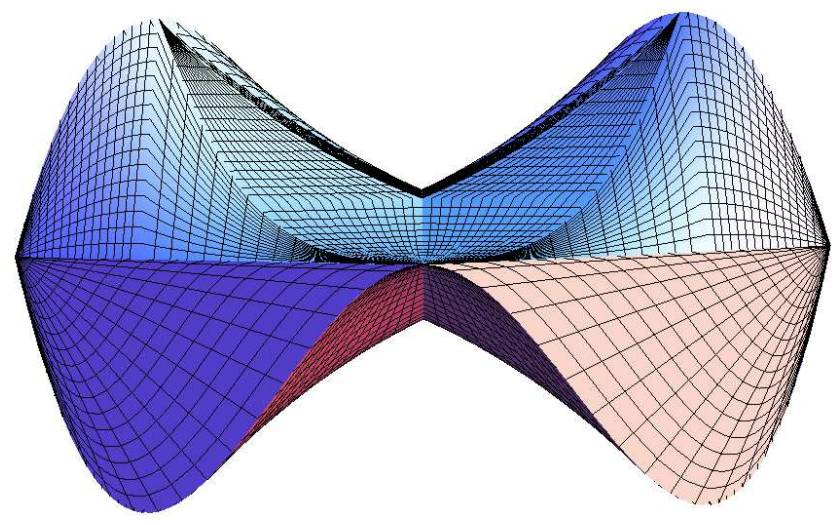

Fig. 10: Unit sphere for the Martinet structure (15). View 1

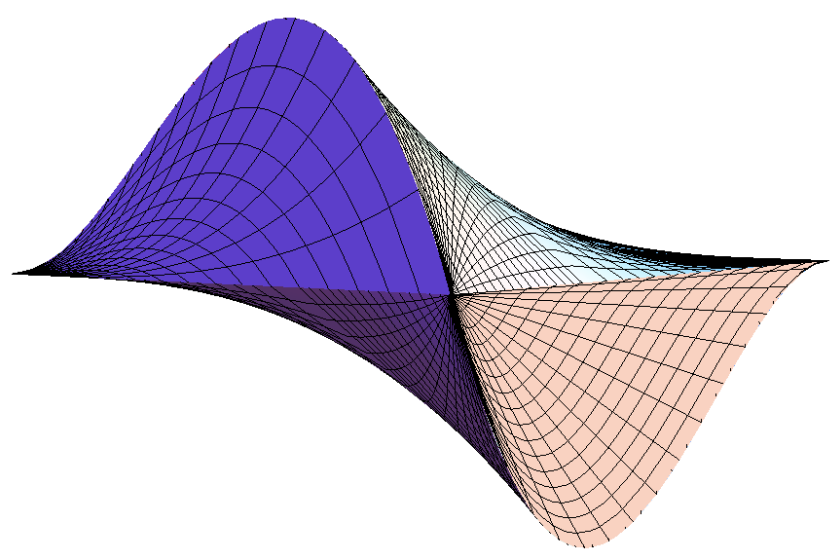

Fig. 11: Unit sphere for the Martinet structure (15). View 2

\section{REFERENCES}

[AG90] A. A. Agrachëv and R. V. Gamkrelidze, Symplectic geometry for optimal control, Nonlinear controllability and optimal control, Monogr. Textbooks Pure Appl. Math., vol. 133, Dekker, New York, 1990, pp. 263-277.

[AS03] Andrei A. Agrachev and Mario Sigalotti, On the local structure of optimal trajectories in $\mathbf{R}^{3}$. SIAM J. Control Optim. 42 (2003), no. 2, 513-531. MR 1982281 (2004f:49042)

[BLD13] Emmanuel Breuillard and Enrico Le Donne, On the rate of convergence to the asymptotic cone for nilpotent groups and subFinsler geometry, Proc. Natl. Acad. Sci. USA 110 (2013), no. $48,19220-19226$.

[BP04] Ugo Boscain and Benedetto Piccoli, Optimal syntheses for control systems on 2-D manifolds, Mathématiques \& Applications (Berlin) [Mathematics \& Applications], vol. 43, SpringerVerlag, Berlin, 2004. MR 2031058 (2005m:49002)

[PBGM83] L. S. Pontryagin, V. G. Boltyanski1, R. V. Gamkrelidze, and E. F. Mishchenko, The Mathematical Theory of Optimal Processes, fourth ed., "Nauka", Moscow, 1983. MR MR719372 (84i:93008)

[Sig05] M. Sigalotti, Local regularity of optimal trajectories for control problems with general boundary conditions, J. Dyn. Control Syst. 11 (2005), no. 1, 91-123. MR 2122468 (2005i:49002) Philippe Souères and Jean-Paul Laumond, Shortest paths synthesis for a car-like robot, IEEE Trans. Automat. Control 41 (1996), no. 5, 672-688. MR 1386992 (97a:70006) 